\title{
Ormone anti-mülleriano (AMH): funzione e livelli nell'uomo e nella donna
}

\author{
Antonio F. Radicioni ${ }^{1}$. Antonella Anzuini ${ }^{1} \cdot$ Francesco Carlomagno $^{1}$
}

Accettato: 2 marzo 2021 / Pubblicato online: 27 maggio 2021

(c) The Author(s) 2021

\section{Introduzione}

L'ormone anti-Mülleriano (AMH) è una glicoproteina appartenente alla superfamiglia del TGF- $\beta$, inizialmente identificato come responsabile della differenziazione dell'apparato genitale maschile.

L'AMH è prodotto come pro-ormone costituito da due monomeri, uniti mediante ponti disolfuro, che subiscono un clivaggio proteolitico a formare due omodimeri: il primo costituito dalle pro-regioni N-terminali e l'altro dalle regioni mature C-terminali. Questi rimangono legati in maniera non-covalente a formare un complesso circolante di 140 $\mathrm{kDa}$.

\section{Metodi analitici}

Attualmente sono disponibili sistemi di dosaggio per la determinazione della concentrazione di AMH che, utilizzando metodiche ELISA a sandwich e anticorpi monoclonali diretti sia contro epitopi della regione matura che della proregione, raggiungono elevata sensibilità (fino a $0,08 \mathrm{ng} / \mathrm{mL}$ con kit di seconda generazione). Tali sistemi sono tuttavia gravati da importanti limitazioni: l'assenza di uno standard internazionale di riferimento per la calibrazione e la mancata comparabilità con i risultati dei precedenti kit, cui si aggiungono dubbi riguardo alla stabilità dell'AMH durante la conservazione e la lavorazione del campione. I valori di riferimento del laboratorio degli autori sono riportati in Tabella 1.

$凶$ F. Carlomagno

francesco.carlomagno@uniroma1.it

1 U.O.D. Diagnostica ormonale, Seminologia e Banca del seme, Policlinico Umberto I, Dipartimento di Medicina Sperimentale, Sezione di Fisiopatologia Medica, Sapienza Università di Roma, Roma, Italia

\section{Valore diagnostico nel sesso maschile}

Nel maschio l'AMH è secreto dalle cellule di Sertoli (CS) immature e, durante la vita fetale, è responsabile della regressione dei dotti di Müller verso l'ottava-nona settimana di gestazione [1]. I livelli di AMH sono relativamente ridotti alla nascita, ma tendono ad aumentare nei primi 6-9 mesi di vita (minipubertà), riflettendo una proliferazione delle CS conseguente all'incremento dei livelli dell'FSH. Le concentrazioni dell'ormone si mantengono relativamente stabili ed elevate durante l'infanzia e la tarda pre-pubertà. All'inizio dello sviluppo puberale, l'incremento del testosterone determina un'ulteriore maturazione/differenziazione delle CS e down-regolazione dell'espressione del gene dell'AMH, cui consegue un rapido e progressivo decremento dei valori dell'ormone sertoliano. Da questa fase, l'AMH viene rilasciato principalmente nel lume dei tubuli seminiferi, con livelli nel plasma seminale maggiori rispetto a quelli sierici. In casi di gonade bilateralmente non palpabile il dosaggio dell'AMH può essere impiegato nella diagnostica differenziale tra il criptorchidismo bilaterale e l'anorchia o la disgenesia gonadica. Inoltre, durante l'infanzia, l'ormone rappresenta un marcatore di funzione sertoliana (insieme all'inibina B); al contrario, dopo la pubertà e nell'uomo adulto il notevole decremento dei valori di AMH non ne consente un impiego diagnostico (Tabella 2).

\section{Valore diagnostico nel sesso femminile}

Nella donna l'AMH è espresso dalle cellule della granulosa (CG) dei piccoli follicoli in sviluppo, dalla fase di reclutamento dei follicoli primordiali fino allo stadio di follicoli pre-antrali e piccoli follicoli antrali (fino a $8 \mathrm{~mm}$ di diametro) [2]. Esso funge da fattore di sopravvivenza per 
Tabella 1 Valori di riferimento dell' AMH sierico in diversi gruppi di soggetti normali. Metodica di riferimento: Beckman Coulter AMH Gen II ELISA. $N D$, non determinabile, inferiore ai limiti di rilevazione della metodica; *, dati degli autori

\begin{tabular}{llll}
\hline Popolazione & $\begin{array}{l}\text { Età, anni } \\
\text { mediana (range) }\end{array}$ & $\begin{array}{l}\text { AMH sierico, ng/mL } \\
\text { mediana }\end{array}$ & $\begin{array}{l}\text { Percentili, ng/mL } \\
2,5^{\circ}-97,5^{\circ}\end{array}$ \\
\hline Bambini pre-puberi $\left(^{*}\right)$ & $5,1(0,3-12)$ & 62,3 & $18,1-186,3$ \\
Bambine pre-pubere & 5,0 & 1,3 & ND-8,9 \\
Uomini puberi e post-puberi $\left(^{*}\right)$ & $36,2(12-68)$ & 3,7 & $0,6-12,8$ \\
Donne in età fertile & - & 1,5 & ND-8,9 \\
Donne in post-menopausa & 71 & $\mathrm{ND}$ & $\mathrm{ND}$ \\
\hline
\end{tabular}

Tabella 2 Valore diagnostico del dosaggio dell'AMH sierico. $N D$, non determinabile; $O H S S$, sindrome da iper-stimolazione ovarica

\begin{tabular}{|c|c|c|c|}
\hline \multicolumn{2}{|l|}{ Uomo } & \multicolumn{2}{|l|}{ Donna } \\
\hline Condizione & AMH sierico e diagnosi differenziale & Condizione & AMH sierico \\
\hline $\begin{array}{l}\text { Gonade non palpabile } \\
\text { bilateralmente }\end{array}$ & $\begin{array}{l}\text { ND: } \\
\text { - Anorchia } \\
\text { - Sindrome dei dotti Mülleriani persistenti } \\
\quad(\text { mutazione del gene } A M H)\end{array}$ & $\begin{array}{l}\text { Valutazione del danno ovarico da } \\
\text { poli-chemioterapia (valori di } \\
\text { AMH prima della chemioterapia) }\end{array}$ & $\begin{array}{l}<0,53 \mathrm{ng} / \mathrm{mL}: \text { predittivo di } \\
\text { amenorrea iatrogena }\end{array}$ \\
\hline & $\begin{array}{l}\text { Basso: } \\
\text { - Ipogonadismo primario } \\
\text { - Ipogonadismo centrale } \\
\text { - (Sindrome da disgenesia testicolare) }\end{array}$ & & $\begin{array}{l}0,53-2,84 \mathrm{ng} / \mathrm{mL} \text { : predittivo di } \\
\text { amenorrea iatrogena/riserva } \\
\text { ovarica preservata in base } \\
\text { all'età (rispettivamente } \\
\text { superiore/inferiore a } 38,6 \text { anni) }\end{array}$ \\
\hline & $\begin{array}{l}\text { Normale: } \\
\text { - Sindrome dei dotti Mülleriani persistenti } \\
\quad(\text { mutazione dell'AMHR) }\end{array}$ & & $\begin{array}{l}>2,84 \mathrm{ng} / \mathrm{mL} \text { : predittivo di } \\
\text { riserva ovarica preservata }\end{array}$ \\
\hline Micropene & $\begin{array}{l}\text { Basso: } \\
\text { - Ipogonadismo primario } \\
\text { - Ipogonadismo centrale } \\
\text { Normale: } \\
\text { - Micropene malformativo }\end{array}$ & $\begin{array}{l}\text { Predizione dell'età alla } \\
\text { menopausa }\end{array}$ & vedi [3] \\
\hline Pubertà ritardata & $\begin{array}{l}\text { Basso: } \\
\text { - Ipogonadismo primario } \\
\text { - Ipogonadismo centrale } \\
\text { Normale: } \\
\text { - Ritardo costituzionale di crescita e pubertà } \\
\quad \text { (RCCP) }\end{array}$ & $\begin{array}{l}\text { Possibile criterio eziologico } \\
\text { nell'insufficienza ovarica } \\
\text { prematura (premature ovarian } \\
\text { failure, POF) }\end{array}$ & $\begin{array}{l}\text { ND: } \\
\text { - POF idiopatica } \\
\text { Basso-normale: } \\
\text { - POF autoimmune } \\
\text { - POF da causa genetica }\end{array}$ \\
\hline $\begin{array}{l}\text { Segni di pubertà } \\
\text { precoce }\end{array}$ & $\begin{array}{l}\text { Basso: } \\
\text { - Pubertà precoce centrale } \\
\text { - Pubertà precoce periferica } \\
\text { Normale: } \\
\text { - Iperplasia surrenalica congenita (ISC) } \\
\text { - Tumore surrenalico androgeno-secernente }\end{array}$ & $\begin{array}{l}\text { Possibile criterio diagnostico } \\
\text { nella sindrome dell'ovaio } \\
\text { policistico (PCOS) }\end{array}$ & $\begin{array}{l}\geq 4,45 \mathrm{ng} / \mathrm{mL} \text { : criterio } \\
\text { surrogato per morfologia } \\
\text { ovarica policistica (PCOM) }\end{array}$ \\
\hline
\end{tabular}

i piccoli follicoli in sviluppo, riducendone la sensibilità all'FSH e la crescita dei follicoli pre-antrali indotta dallo stesso. Agisce, inoltre, da regolatore della produzione estro- genica follicolare: assicura così che ciascun piccolo follicolo antrale produca bassi livelli di estradiolo prima della selezione. 
Tabella 2 (Continued)

\begin{tabular}{|c|c|c|c|}
\hline \multicolumn{2}{|l|}{ Uomo } & \multicolumn{2}{|l|}{ Donna } \\
\hline Condizione & AMH sierico e diagnosi differenziale & Condizione & AMH sierico \\
\hline $\begin{array}{l}\text { Disordini dello sviluppo } \\
\text { sessuale (DSD) }\end{array}$ & $\begin{array}{l}\text { ND: } \\
\text { - 46, XY disgenesia gonadica completa } \\
\text { Basso: } \\
\text { - 46, XY disgenesia gonadica parziale } \\
\text { - Sindrome di Klinefelter (47, XXY) } \\
\text { - Aneuploidie maggiori dei cromosomi ses- } \\
\text { suali (HGA) } \\
\text { - DSD ovotesticolare } \\
\text { Normale: } \\
\text { - Difetto di sintesi degli androgeni } \\
\text { - Sindrome da insensibilità agli androgeni } \\
\text { - 46, XY DSD malformativo } \\
\text { - 46, XX maschio (DSD testicolare) }\end{array}$ & $\begin{array}{l}\text { Individualizzazione della terapia } \\
\text { e prognosi in donne sottoposte a } \\
\text { fertilizzazione in vitro (IVF) } \\
\text { (valori di AMH prima della } \\
\text { stimolazione ovarica) }\end{array}$ & $\begin{array}{l}\leq 1,26 \mathrm{ng} / \mathrm{mL} \text { : predittivo di } \\
\text { normale risposta a } \\
\text { iper-stimolazione ovarica } \\
\text { controllata }(\mathrm{COH}) \\
>3,36 \mathrm{ng} / \mathrm{mL} \text { : predittivo di } \\
\text { OHSS in seguito a COH } \\
<4,75 \mathrm{ng} / \mathrm{mL} \text { : predittivo di } \\
\text { risposta ovulatoria a un } \\
\text { primo ciclo di COH in donne } \\
\text { affette da PCOS }\end{array}$ \\
\hline
\end{tabular}

Nella donna si osserva un picco nei livelli sierici di AMH poco dopo la nascita che, nel tempo, aumenta progressivamente fino a raggiungere un picco intorno ai 25 anni di età, seguito da un costante declino fino a livelli non più rilevabili all'inizio della menopausa [3].

Sebbene i livelli di AMH mostrino un'elevata variabilità inter-individuale nella donna, la variabilità intra-individuale è funzione principalmente dell'età, con fluttuazioni minime durante il ciclo mestruale. Una riduzione significativa si osserva, tuttavia, in seguito a soppressione ovarica mediante interventi farmacologici o durante la gravidanza.

I valori di AMH (Tabella 2) sono utilizzati per predire la risposta ovarica e personalizzare la terapia durante le procedure di fecondazione in vitro, nella valutazione del danno della riserva follicolare ovarica indotto da fonti iatrogene, quali radio- o chemioterapia, per predire l'età di insorgenza della menopausa, quale possibile criterio diagnostico nella sindrome dell'ovaio policistico (PCOS) ed eziologico nell'insufficienza ovarica prematura (POF).

\section{Conclusioni}

Recenti avanzamenti nelle metodiche di dosaggio dell'AMH e una migliore comprensione della sua fisiopatologia ne hanno rafforzato il ruolo come marcatore di funzione gonadica. Nel maschio la determinazione dei livelli di AMH si rende utile soprattutto nella diagnosi differenziale in caso di gonade non palpabile bilateralmente, genitali ambigui o nel sospetto di disordini dello sviluppo sessuale (DSD); al contrario, nella donna il suo utilizzo è incentrato alla valutazione della riserva ovarica (anche conseguente a danno ovarico iatrogeno), oltre che alla predizione della risposta alla stimolazione ovarica e come criterio surrogato di morfologia ovarica policistica (PCOM).

Funding Note Open access funding provided by Università degli Studi di Roma La Sapienza within the CRUI-CARE Agreement.

Conflitto di interesse Gli autori Antonio F. Radicioni, Antonella Anzuini e Francesco Carlomagno dichiarano di non avere conflitti di interesse.

Consenso informato Lo studio presentato in questo articolo non ha richiesto sperimentazione umana.

Studi sugli animali Gli autori di questo articolo non hanno eseguito studi sugli animali.

Nota della casa editrice Springer Nature rimane neutrale in riguardo alle rivendicazioni giurisdizionali nelle mappe pubblicate e nelle affiliazioni istituzionali.

Open Access This article is licensed under a Creative Commons Attribution 4.0 International License, which permits use, sharing, adaptation, distribution and reproduction in any medium or format, as long as you give appropriate credit to the original author(s) and the source, provide a link to the Creative Commons licence, and indicate if changes were made. The images or other third party material in this article are included in the article's Creative Commons licence, unless indicated otherwise in a credit line to the material. If material is not included in the article's Creative Commons licence and your intended use is not permitted by statutory regulation or exceeds the permitted use, you will need to obtain permission directly from the copyright holder. To view a copy of this licence, visit http://creativecommons.org/licenses/by/4.0/. 


\section{Bibliografia}

1. Edelsztein NY, Grinspon RP, Schteingart HF, Rey RA (2016) AntiMüllerian hormone as a marker of steroid and gonadotropin action in the testis of children and adolescents with disorders of the gonadal axis. Int J Pediatr Endocrinol 20:1-10
2. Moolhuijsen LM, Visser JA (2020) Anti-Müllerian hormone and ovarian reserve: update on assessing ovarian function. $\mathrm{J}$ Clin Endocrinol Metab 105(11):3361-3373

3. Tehrani FR, Solaymani-Dodaran M, Tohidi M et al (2013) Modeling age at menopause using serum concentration of Anti-Mullerian Hormone. J Clin Endocrinol Metab 98(2):729-735 